\title{
Microstructure and macrostructure
}

Musical coherence is essential for the achievement of meaning in any particular work. While it is possible to speculate on a variety of reasons which may facilitate this coherence, many would agree that an important determinant may be found in the successful articulation of the work's structure. Furthermore, structure is realised through discourse, which is dependent on the articulation of actual sounds: inflections, accents, local changes in tempo, etc., may alter the meaning of a passage significantly. Although this is true of any music, it acquires additional significance for the composer in the electroacoustic domain since she becomes responsible for the inner attributes of timbre.

It is therefore not surprising that the structure of a significant number of electroacoustic works is, in one way or another, abstracted from the characteristics of their materials. The important relationship between small temporal units - such as motives and phrases - and overall form of a piece is extended to encompass the inner mechanisms of the constituents of those units: the sounds themselves. Furthermore, changes in the conception of the world, such as the idea of self-similarity, with its projection of structure from the microscopic to the macroscopic, have also had an effect on the way musicians think.

However, the questions remaining unanswered are numerous. For instance, can there be structure which unifies a work at different temporal levels? Can this unification include timbre so that the inner characteristics of its sounds affect the structure of a piece? Could time be completely absorbed by timbre to an extent which includes the overall temporal span of a work - emulating the integration of space and time in the theory of relativity? It is possible to preprogram this type of cross-dimensional unity? If so, how? On the other hand, is it possible, perhaps, that the above pursuits are nothing but a dangerous chimera which diverts the efforts and endeavours of composers towards a formal vacuum devoid of meaning?

The articles in this issue respond to some of these and other related questions and, at the same time, formulate new queries of their own. The first two present different types of pragmatic approach towards the achievement of unity at various temporal and timbral levels. Robert Newcomb's description of the philosophy behind the implementation of the MITA software system advocates unity without preconceptions. MITA relies on processes which seek to establish a strong relationship between materials and overall structure, learning the attributes and interrelations of the former and employing these as the basis for the establishment of the latter. It also aims to assimilate human experience within the structure of the work by increasing its knowledge databases, and by taking into account environmental parameters and real-world events. Christos Hatzis, on the other hand, discusses the strategies employed in the creation of a specific work entitled The Law of One, in which he aimed to achieve a holistic integration and unification of compositional parameters out of those of a single sound, based on an original approach to the harmonic series.

Hatzis' pragmatic presentation is contrasted by Mladen Milicevic's questioning of the validity of structure as sole conveyer of musical meaning. In his view, the importance attributed to the former is overwhelming insofar as there is no true objectivity in music. He then proposes a postmodern alternative which relies on the perpetuation of ideas within culture in an analogous manner to that of genes in the evolutionary process, as encapsulated in Richard Dawkin's concept of meme.

Microstructure and macrostructure are further alluded to in the context of general aesthetic and philosophical considerations: Carlos Palombini examines the status of musique concrete, in view of the spectre raised by the modern relationship between human beings and technology-which prompted Heidegger's call for a return to the Greek conception of techne-and the implications of instant and mass reproduction of art objects - which Benjamin terms 'loss of aura'. He proposes the possibility of redemption inherent in the attention to the microstructure of sounds and its implications and associations regarding the overall structure of a piece encapsulated in Schaeffer's concept of the 'sonic object' identified by 'reduced listening'.

Francesco Giomi and Marco Ligabue present an analytical view, aiming to establish the existence of 
a set of strategies which shape and give meaning to discourse in six seminal early electroacoustic works. The search for events - or combinations of events which attain coherent musical sense is carried out at various levels, from the overall structure to individual sounds.

The last allusion to microstructure and macrostructure comes from Tsippi Fleischer's response to this theme, as a composer who concentrates on various structural aspects of two of her works. This discussion includes the use of microtones and the phonetic qualities of the Arabic language in the cantata Like Two Branches, and the classification of speech material according to its timbral qualities in order to achieve a metaphor for the transition from night to day throughout the duration of the tape piece The Gown of Night.

The Tutorial Article by Giovanni De Poli and Davide Rochesso provides a comprehensive overview of the applications of physical modelling in the realisation of sound sources and signal processing, and in their integration with physics-based graphic models. It is accompanied by an exhaustive list of references. Victor Lazzarini discusses the design, current state of development, and future improvements of a synthesis and processing system motivated by pedagogical concerns which aims to provide a variety of options in a simple and intuitive way.

Finally, it is worth calling the attention of the reader to the international flavour of this issue, which includes articles from authors in five different countries. It is therefore hoped that, in addition to being testimony to a diversity of views and conceptions held in the music community, this issue reflects the fact that interest in the matters under discussion extends beyond geographical distance and demarcation.

Rajmil Fischman 